\title{
Estudo sobre dados relevantes para o acompanhamento de participações em jogos educacionais
}

\author{
Frederico C. Cardoso ${ }^{1}$, Joice L. Otsuka ${ }^{1}$, Miguel S. Tosta ${ }^{1}$, Delano M. Beder ${ }^{1}$ \\ ${ }^{1}$ Departamento de Computação - Universidade Federal de São Carlos (UFSCar) \\ Rod. Washington Luís - km 235 - SP-310 - 13565-905 - São Carlos - SP - Brasil \\ \{frederico.cardoso, joice, miguel, delano\}@ufscar.br
}

\begin{abstract}
The use of games in the learning process can be a very interesting alternative and can provide several benefits for the students, but its use is still limited due to several difficulties encountered by the teachers. This work presents a survey carried out with the teachers with the objective of analyzing their real difficulties, needs and objectives in the use of educational games in their classes, as well as, what are the information considered most relevant to the monitoring and performance analysis of their students.
\end{abstract}

Resumo. O uso de jogos no processo de aprendizado pode ser uma alternativa bastante interessante, além de proporcionar diversos benefícios para os alunos. Entretanto seu uso ainda é limitado, devido a diversas dificuldades encontradas pelos professores, sobretudo no acompanhamento dos alunos. Este trabalho apresenta um levantamento realizado junto com professores do ensino fundamental, médio, técnico e superior com o objetivo de analisar suas reais dificuldades, necessidades e objetivos no uso de jogos educacionais em suas aulas, bem como, um modelo genérico de acompanhamento que pode ser aplicado em diferentes tipos de jogos educacionais, com os indicadores de acompanhamento considerados mais relevantes para a análise de desempenho de seus alunos.

\section{Introdução}

Os jogos educacionais têm ganhado grande destaque como recursos de apoio aos processos educacionais, por estarem mais alinhados com os estilos de aprendizagem das gerações que nasceram e cresceram imersas nas tecnologias digitais. Prensky (2012) aponta as mudanças no estilo cognitivo dos ditos "nativos digitais", dentre as quais destacam-se: o processamento de informações em uma velocidade mais alta; a facilidade de realizar várias atividades em paralelo; a preferência por gráficos a textos; a preferência por acesso aleatório e não sequencial; além de serem extremamente conectados, ativos e fluentes em tecnologias. De acordo com Squire (2007), "a aprendizagem baseada em jogos é uma forma de engajar os alunos em atividades significativas e permitir que assumam novas identidades, explorem mundos e aprendam".

Entretanto, apesar de os jogos poderem proporcionar diversos benefícios para os alunos, alguns professores ainda são resistentes ao uso desses recursos educacionais, principalmente pela dificuldade de acompanhamento do que acontece enquanto os alunos estão jogando. Alonso-Fernandez et al. (2017) apontam algumas dessas dificuldades, como: diferentes objetivos de aprendizagem e tipos de análise; diferentes níveis de interesse e motivação entre os alunos; diferentes tipos de jogos educacionais; e quais dados devem ser coletados e como apresentar esses dados. 
VIII Congresso Brasileiro de Informática na Educação (CBIE 2019)

Anais do XXX Simpósio Brasileiro de Informática na Educação (SBIE 2019)

Este trabalho visa investigar o potencial e limitações do uso de técnicas de Visualização de Informação (InfoVis) para o acompanhamento do progresso de estudantes em suas interações com jogos educacionais.

A área de InfoVis pode ser definida como a exibição gráfica de informações abstratas que possui duas finalidades: análise de dados e comunicação (FEW, 2013). O objetivo básico e principal relacionado à visualização é poder extrair o máximo de informações possíveis a partir de um conjunto de dados coletados (CARVALHO; MARCOS, 2009).

Um modelo básico para a criação de visualizações possui três etapas principais: (i) Transformação de dados; (ii) Mapeamento visual e (iii) Transformações de visões (CARD; MACKINLAY; SHNEIDERMAN, 1999). Este trabalho aborda estas três etapas e tem como objetivo apresentar um modelo genérico de rastreamento de interações em jogos que colabore com a coleta de dados e geração de representações visuais que atendam as necessidades de análise, acompanhamento e avaliação de alunos por parte dos professores. Pretende-se dessa forma, trazer contribuições para a área de InfoVis aplicada à jogos educacionais.

Os seguintes procedimentos e métodos foram utilizados na condução dessa pesquisa: (i) mapeamento sistemático da literatura com o intuito de identificar os principais trabalhos relacionados a InfoVis em jogos; (ii) aplicação de um survey com professores que utilizam jogos em suas práticas educacionais, para o levantamento de seus objetivos de acompanhamento e suas dificuldades; (iii) definição de um conjunto de dados que deve ser coletado dos jogos para que seja possível atender os indicadores de acompanhamento definidos; (iv) definição de um conjunto de representações visuais mais adequadas para representar graficamente cada um dos indicadores de acompanhamento; e (v) desenvolvimento e apresentação de uma Dashboard de acompanhamento composta pelo conjunto de representações visuais definido.

Para a realização e avaliação das atividades deste estudo foram utilizados jogos educacionais disponíveis na plataforma REMAR ${ }^{1}$, plataforma que permite a criação de diferentes instâncias de jogos educacionais com conteúdos customizados pelos professores, desenvolvida no Laboratório de Objetos de Aprendizagem (LOA) da UFSCar.

O restante do artigo está organizado da seguinte forma: a seção 2 apresenta os principais trabalhos relacionados. Na seção 3 são apresentados os principais resultados do survey aplicado aos professores, bem como o conjunto de indicadores definido. $\mathrm{Na}$ seção 4 é apresentado o conjunto de dados a ser coletado dos jogos educacionais. Na seção 5, é apresentado o conjunto de representações visuais. Na seção 6, é apresentada a Dashboard de acompanhamento proposta. Na seção 7 são apresentados os principais resultados da validação inicial de toda a solução proposta. Na seção 8 são apresentadas as conclusões e trabalhos futuros.

\section{Trabalhos relacionados}

Dos trabalhos obtidos a partir do mapeamento sistemático (CARDOSO; OTSUKA, 2018), dois são bastante relacionados com a nossa pesquisa. Alonso-Fernandez et al. (2017) descrevem o processo para a sistematização do Game Learning Analytics (GLA), através de dois passos: (i) um modelo de rastreamento de informações padrão e (ii)

\footnotetext{
${ }^{1}$ http://www.loa.sead.ufscar.br/
} 
VIII Congresso Brasileiro de Informática na Educação (CBIE 2019)

Anais do XXX Simpósio Brasileiro de Informática na Educação (SBIE 2019)

análise e visualização padrão para gerar informações em qualquer Serious Game (SG) que siga o modelo de rastreamento proposto. Os autores apontam ainda a possibilidade de personalização e adaptação deste modelo para determinados jogos, caso seja necessário. Ao final do estudo, os autores propõem a visualização de um conjunto de informações que eles consideram úteis para uma ampla variedade de possíveis grupos de interesse.

Serrano-Laguna \& Fernández-Manjón (2014) apresentam uma abordagem que busca simplificar a tarefa dos professores ao usarem jogos em sala de aula, proporcionando uma visualização orientada a objetivos das informações de seus alunos em tempo real, enquanto utilizam os jogos. Foram destacados três aspectos principais: definir objetivo educacional dos exercícios, estabelecer uma conexão entre interações do jogo e os objetivos e projetar visualizações para fornecer informações úteis para os professores. Os autores concluem que a análise requer técnicas simples e que as visualizações orientadas por objetivos ajudaram o professor a ter uma visão mais completa do desempenho dos alunos.

Os trabalhos mencionados acima apresentam soluções que visam melhorar o processo de acompanhamento e análise de interações em jogos educacionais, mas estes ainda encontram algumas limitações. O primeiro trabalho apresenta um conjunto de informações que são coletadas e analisadas em jogos educacionais, mas não há informações sobre os métodos utilizados para a identificação dos dados relevantes para o acompanhamento, não havendo uma validação desse conjunto. O segundo propõe uma método para desenvolvimento de jogos educacionais baseada nos objetivos de avaliação de cada professor, porém, os resultados apresentam soluções desenvolvidas especificamente para atender cada professor. Desta forma, o trabalho aqui apresentado visa contribuir com uma solução mais genérica analisando as principais demandas de acompanhamento relevantes para o público-alvo. Para o levantamento dessas demandas, foi conduzida uma pesquisa survey que é apresentada na seção seguinte.

As próximas seções apresentam os principais resultados da solução proposta neste estudo.

\section{Conjunto de indicadores de acompanhamento}

Com o intuito de conhecer os objetivos dos professores no uso e acompanhamento de jogos educacionais, bem como suas dificuldades, foi conduzida uma pesquisa survey com professores do ensino fundamental, médio, técnico e superior que utilizam jogos em suas práticas educacionais. Foram convidados professores que participam de projetos piloto com jogos educacionais da plataforma REMAR, além de professores do ensino superior que usam jogos e/ou pesquisam sobre jogos educacionais.

Os professores foram convidados a responder um questionário online, primeiramente, para verificar seus dados pessoais, há quanto tempo e em qual nível de ensino lecionam. Dos 37 professores que aceitaram responder o questionário, 15 lecionam somente no ensino superior, 13 somente no ensino fundamental, 4 nos ensinos fundamental e médio, 2 somente no ensino médio, 2 nos ensinos fundamental e superior e 1 nos ensinos fundamental e técnico. Além disso, 4 estão nos primeiros 5 anos de carreira, enquanto que a maioria (24 dos 37 ) leciona há mais de 15 anos.

De todos os professores que responderam o questionário, apenas dois nunca utilizaram jogos educacionais no processo de aprendizagem de seus alunos. A frequência de 
VIII Congresso Brasileiro de Informática na Educação (CBIE 2019)

Anais do XXX Simpósio Brasileiro de Informática na Educação (SBIE 2019)

uso é mostrada na Figura 1.

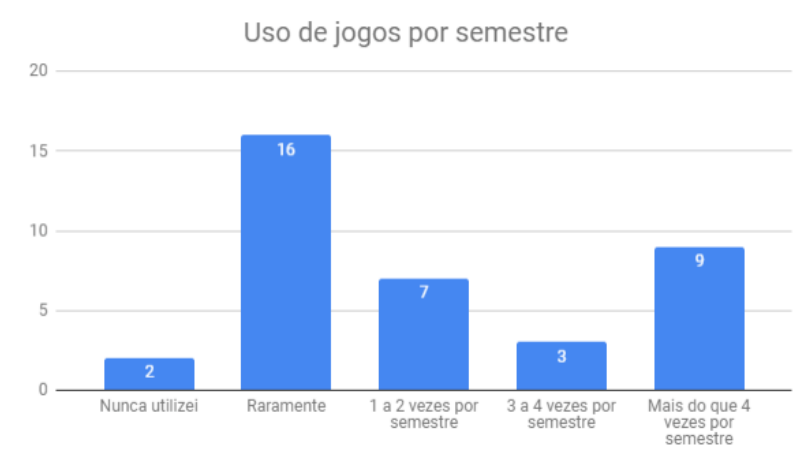

Figura 1. Número de vezes que o professor utiliza jogos por semestre

A primeira questão analisada no survey foi relacionada aos objetivos dos professores participantes no uso dos jogos educacionais. Os principais objetivos identificados foram: (a) analisar o potencial do jogos na mediação da aprendizagem; (b) explorar a ludicidade dos jogos como fator motivacional para a aprendizagem; (c) explorar a interatividade e a possibilidade de simular situações reais em um ambiente seguro e interativo; (d) promover uma estimuladora e significativa; (e) envolver os alunos e aumentar o interesse destes em aprender; (f) treinar/reforçar conceitos e habilidades aprendidos em aula; (g) estimular o raciocínio lógico; e (h) observar e analisar os processos linguísticos e cognitivos que estão envolvidos na interação com jogos.

Para verificar se os objetivos de uso foram alcançados, é preciso realizar um acompanhamento com os alunos. Sendo assim, solicitamos aos professores participantes que indicassem como realizam o acompanhamento de seus alunos durante o uso de jogo. A maioria dos professores indicou como forma de acompanhamento apenas a observação do uso. Alguns indicaram também discussões em grupo, feedback dos alunos por meio de formulários ou discussões, gravação das interações para posterior análise. Em alguns casos os professores aplicam testes ou atividades práticas para avaliar os conhecimentos e habilidades do aluno após o uso dos jogos. Alguns ainda não realizam qualquer tipo de acompanhamento ou avaliação. Nenhum professor indicou algum tipo de acompanhamento por meio da coleta de dados nos jogos durante o uso destes por seus alunos.

Também solicitamos aos professores que nos indicassem os seus objetivos de acompanhamento dos alunos nos jogos, bem como as dificuldades encontradas nesse acompanhamento. A maioria indicou como objetivos: (a) analisar a efetividade da aprendizagem; (b) acompanhar o rendimento; (c) acompanhar o progresso dos alunos; (d) identificar as dificuldades e tirar dúvidas; (e) apoiar o desenvolvimento do conhecimento. Dentre as principais dificuldades no acompanhamento, ou seja, o que gostariam de conseguir acompanhar mas não conseguem, foram apontados: (a) acompanhar a passagem de fases e números de acertos e erros; (b) número de tentativas até chegar ao final; (c) acompanhamento individual dos desafios e soluções de cada aluno; (d) observar o caminho percorrido (tentativas) para analisar o raciocínio usado; (e) analisar como o aluno se sente dentro do jogo; e (f) "acompanhar o que acontece dentro do jogo".

A partir dos trabalhos apresentados na Seção 2, em conjunto com experiências anteriores de nosso grupo de pesquisa e buscando atender as necessidades de acompa- 
VIII Congresso Brasileiro de Informática na Educação (CBIE 2019)

Anais do XXX Simpósio Brasileiro de Informática na Educação (SBIE 2019)

nhamento apontadas pelos professores, foi possível a identificação de um conjunto inicial de indicadores supostamente relevantes para o acompanhamento das participações dos alunos em jogos educacionais. Com o intuito de validar e complementar esse conjunto, o apresentamos aos professores participantes do survey e solicitamos que indicassem a relevância ou não de cada um dos indicadores apresentados. A lista de indicadores com a quantidade de votos pode ser vista na Tabela 1.

Tabela 1. Indicadores de participação a serem coletados dos jogos educacionais

\begin{tabular}{l|l}
\hline Indicadores & Votos \\
\hline Progresso dos alunos por nível (tentativas/conclusão) & $30(81,1 \%)$ \\
\hline Progresso dos alunos por desafio (tentativas/erros) & $30(81,1 \%)$ \\
\hline Número de alunos que concluíram o jogo & $29(78,4 \%)$ \\
\hline Taxa de erro por desafio & $27(73 \%)$ \\
\hline Número de tentativas de concluir cada nível & $26(70,3 \%)$ \\
\hline Tempo de conclusão do jogo & $24(64,9 \%)$ \\
\hline Frequência de respostas por desafio & $22(59,5 \%)$ \\
\hline Número de alunos em cada nível do jogo & $22(59,5 \%)$ \\
\hline Número de tentativas de concluir cada desafio & $21(56,8 \%)$ \\
\hline Tempo de conclusão de cada desafio & $21(56,8 \%)$ \\
\hline Tempo de conclusão de cada nível & $19(51,4 \%)$ \\
\hline Ranking de pontuação & $17(45,9 \%)$ \\
\hline
\end{tabular}

Também solicitamos que os participantes analisassem se o conjunto indicado é suficiente para apoiar o acompanhamento dos alunos nos jogos. De acordo com a maioria dos professores ( 25 dos 37 ), o conjunto foi considerado suficiente para um bom acompanhamento de seus alunos no uso dos jogos. Alguns indicaram a necessidade de um modelo de acompanhamento que fornecesse indicadores da motivação, o índice de satisfação e o fator emocional dos alunos enquanto jogam.

A partir deste etapa identificamos as demandas de professores que atuam em diferentes níveis de ensino e definimos um conjunto inicial de indicadores relevantes para o acompanhamento dos estudantes nos jogos.

\section{Conjunto básico de dados}

O objetivo principal desta etapa, foi definir um conjunto de atributos comuns que devem ser coletados durante a interação dos estudantes com os jogos com o intuito de possibilitar as análises dos indicadores de acompanhamento definidos na seção anterior.

Foram considerados no estudo três jogos disponíveis na plataforma REMAR: o Responda se Puder, um jogo de perguntas e respostas customizáveis e que devem ser respondidas para que seja possível avançar de fase; o Escola Mágica, um jogo de plataforma com quizzes customizáveis que devem ser respondidos para que o jogador possa liberar novas fases; o Em Busca do Santo Grau, jogo modular com fases que podem ser combinadas e customizadas de acordo com os objetivos do professor. Cada fase deste jogo possui recursos e desafios diversificados (por exemplo: na Fase da Tecnologia, é possível compartilhar um vídeo e o desafio está relacionado a adivinhar palavras-chave 
VIII Congresso Brasileiro de Informática na Educação (CBIE 2019)

Anais do XXX Simpósio Brasileiro de Informática na Educação (SBIE 2019)

definidas pelo professor; na Fase da Galeria o desafio é ordenar quadros de acordo com uma sequência que soluciona o caso definido pelo professor; nas fases do Blocos de Gelo, Campo Minado e TCC os jogadores devem responder quizzes definidos pelo professor e evitar sofrer os danos de cada fase.

O conjunto de dados coletados durante a interação dos estudantes e seus significados é apresentado na Tabela 2.

Tabela 2. Dados coletados nos jogos

\begin{tabular}{l|l}
\hline Dados coletados & Descrição \\
\hline gameId & Usado para informar qual é a instância de jogo analisada. \\
\hline userId & Usado para trazer o código do usuário. \\
\hline levelId & Usado para indicar o número do nível. \\
\hline challengeId & Usado para indicar o número do desafio. \\
\hline win & Usado para indicar se o usuário acertou ou errou o desafio. \\
\hline answer & Indica a resposta (escolha) do usuário. \\
\hline score & Indica a pontuação atingida pelo usuário ao final do jogo. \\
\hline time & Usado para indicar o tempo gasto no jogo, nível ou desafio (0=início, $>0=$ conclusão). \\
\hline timeType & $\begin{array}{l}\text { Usado em conjunto com a variável “time”para identificar se o tempo é referente ao } \\
\text { jogo(0), nível(1) ou desafio(2). }\end{array}$ \\
\hline
\end{tabular}

É importante ressaltar que o objetivo foi definir um conjunto mínimo de dados a ser coletado e que cada um desses dados busca atender o máximo de indicadores possível.

\section{Conjunto de representações visuais e framework de desenvolvimento}

Esta etapa foi conduzida em dois passos: (i) definição de um conjunto de representações visuais a ser utilizado para a apresentação dos indicadores; e (2) escolha do framework a ser utilizado para implementação das visualizações.

Em posse dos indicadores apresentados na Seção 3 e os dados a serem coletados apresentados na Seção 4, partimos então para o processo de "Mapeamento Visual". Neste momento, definiu-se quais representações visuais seriam utilizadas para representar graficamente cada um dos indicadores de acompanhamento, de maneira efetiva.

O framework utilizado para a implementação do conjunto de representações visuais, foi o Google Charts $^{2}$, desenvolvido e mantido pela própria Google. Embora outros frameworks tenham sido encontrados, como AnyCharts ${ }^{3}$, Charts.js ${ }^{4}$, Vis.js ${ }^{5}$, Chartist.js ${ }^{6}$; o framework escolhido se destaca pela sua versatilidade, com um conjunto variado de gráficos de fácil implementação e customização, além disso, é um framework que está em constante atualização.

O framework selecionado não é uma "regra" em nosso modelo, ou seja, outros frameworks poderiam ser utilizados.

\footnotetext{
${ }^{2}$ https://developers.google.com/chart/interactive/docs/quick_start

${ }^{3}$ https://anychart.com/pt/products/anychart/overview/

${ }^{4}$ https://chartjs.org/docs/latest/

${ }^{5}$ http://visjs.org/\#download_install

${ }^{6}$ https://gionkunz.github.io/chartist-js/getting-started.html
} 
VIII Congresso Brasileiro de Informática na Educação (CBIE 2019)

Anais do XXX Simpósio Brasileiro de Informática na Educação (SBIE 2019)

\section{Dashboard de acompanhamento}

O desenvolvimento da Dashboard integrando todos os indicadores, seguiu a definição trazida por Few (2013), onde é dito que uma Dashboard "é uma exibição visual das informações mais importantes necessárias para atingir um ou mais objetivos; consolidada e organizada em uma única tela para que as informações possam ser monitoradas rapidamente".

Levando-se em consideração o grande número de indicadores levantados (Seção 3), optou-se por montar e dividir a Dashboard em três níveis de visualizações: indicadores referentes ao jogo de um modo geral; indicadores referentes às fases do jogo; e indicadores referentes aos desafios de cada fase. Estas visualizações possuem dois tipos de visões: dados de toda a turma e dados de cada aluno individualmente.

$\mathrm{Na}$ visão de toda a turma, os indicadores do primeiro nível de visualização (Figura 2), são: (a) Número de alunos que concluíram o jogo e Ranking de pontuação; (b) Tempo de conclusão do jogo; (c) Número de alunos em cada fase do jogo; (d) Número de tentativas de concluir cada fase do jogo; e (e) Tempo de conclusão de cada fase do jogo.

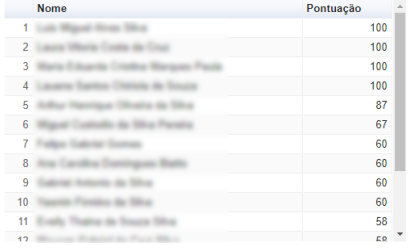

(a)

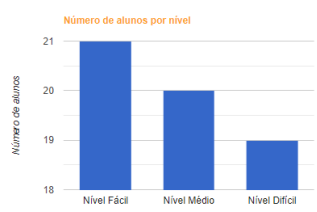

(c)

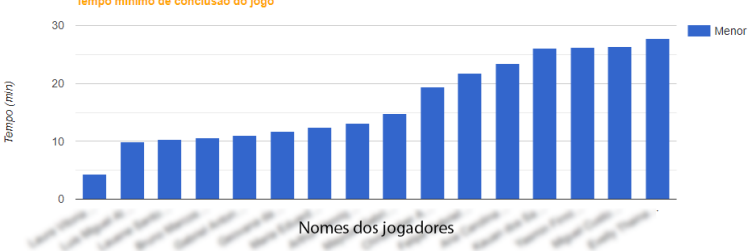

(b)

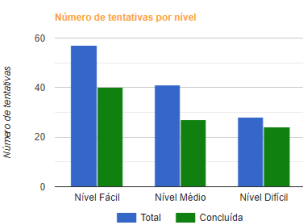

(d)

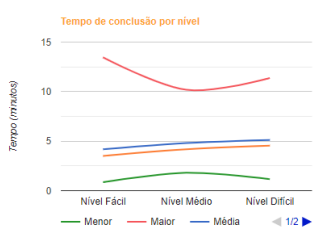

(e)

Figura 2. Indicadores relacionados ao jogo

Os indicadores do segundo nível de visualização (Figura 3), são: (a) Progresso do alunos por fase (todos os alunos presente no nível selecionado, com o número de tentativas e conclusão de cada um); (b) Taxa de erro por desafio; (c) Número de tentativas de concluir cada desafio; e (d) Tempo de conclusão de cada desafio.

Os indicadores do terceiro nível de visualização (Figura 4), são: (a) Progresso dos alunos por desafio (alunos que responderam o desafio selecionado, com o número de erros e acertos de cada um); (b) Frequência de escolhas por desafio (alternativas escolhidas em perguntas múltipla escolha, respostas para palavras embaralhadas, etc.).

Na visão de cada aluno, os indicadores do primeiro nível de visualização (Figura 5), são: (a) Número de tentativas de concluir cada fase; e (b) Tempo de conclusão de cada fase. Já os indicadores do segundo nível de visualização (Figura 6), são: (a) Número de tentativas de concluir cada desafio; (b) Desafios com maior taxa de erros; e (c) Tempo de conclusão de cada desafio. 
VIII Congresso Brasileiro de Informática na Educação (CBIE 2019)

Anais do XXX Simpósio Brasileiro de Informática na Educação (SBIE 2019)

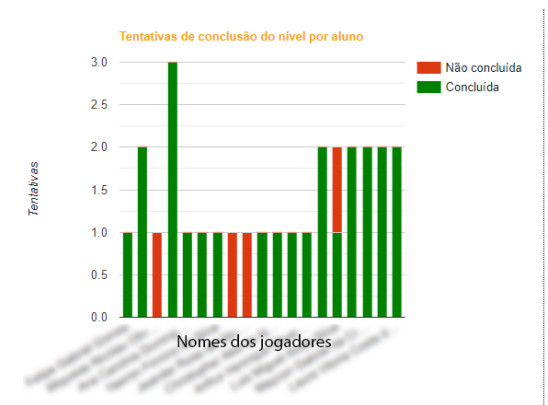

(a)

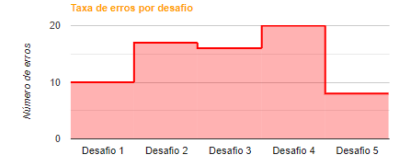

(b)

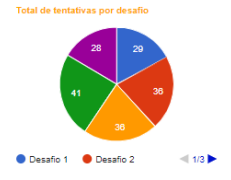

(c)

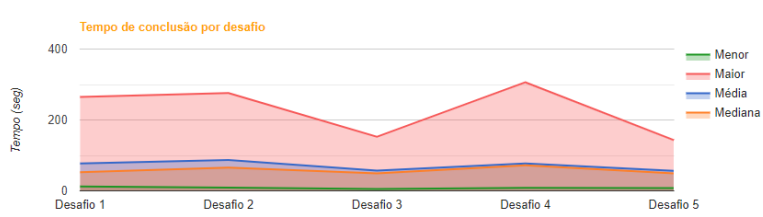

(d)

Figura 3. Indicadores relacionados a cada fase do jogo

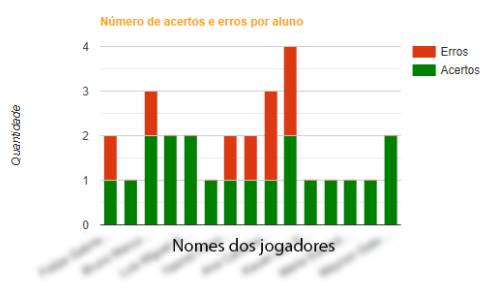

(a)

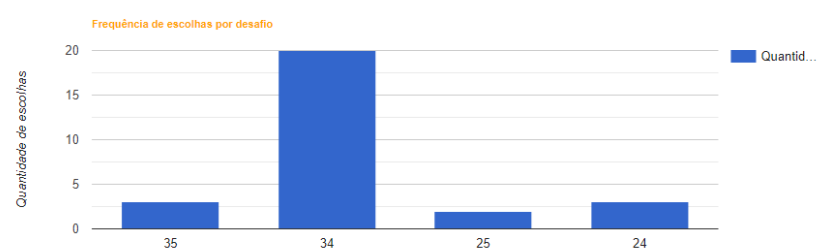

(b)

Figura 4. Indicadores relacionados aos desafios de cada fase do jogo

\section{Validação inicial do modelo proposto}

Buscando validar a solução proposta, 4 professores foram convidados a aplicar um jogo com sua turma, analisar a Dashboard e responder um questionário online, dividido em 5 partes: (i) identificação do participante; (ii) validação do conjunto de indicadores; (iii) validação do conjunto de representações visuais; (iv) validação da estrutura da Dashboard; e (v) validação da usabilidade da Dashboard.

Todos os quatro professores são experientes e fazem uso de jogos educacionais em seu processo de ensino, em disciplinas como Português, Matemática, Geografia, História e Ciências.

As respostas para as perguntas do questionário utilizavam uma escala Likert de 5 níveis, onde 1 significa "Discordo totalmente" e 5 significa "Concordo totalmente".

Para o conjunto de indicadores, perguntamos aos professores se este era suficiente para o acompanhamento das interações de seus alunos nos jogos, e todos eles respon-

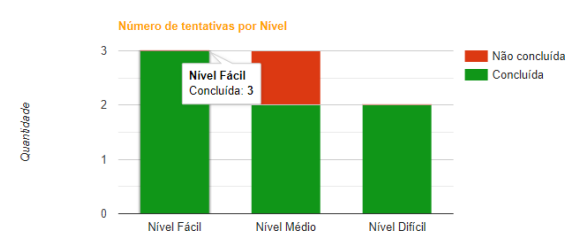

(a)

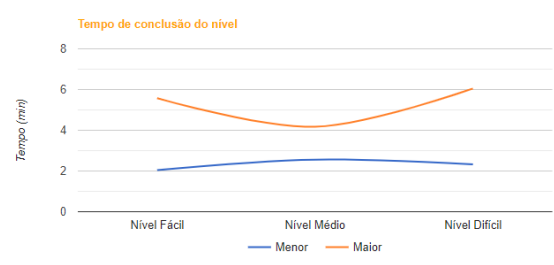

(b)

Figura 5. Indicadores relacionados a cada fase do jogo 


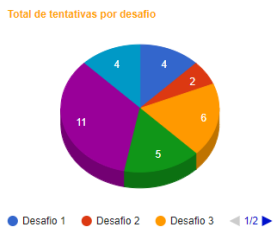

(a)

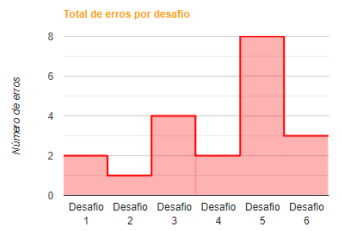

(b)

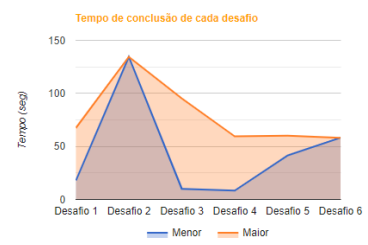

(c)

Figura 6. Indicadores relacionados aos desafios de cada fase do jogo

deram de modo positivo, sendo que 3 concordam totalmente e 1 parcialmente. Também perguntamos se algum indicador deveria ser retirado ou acrescentado ao conjunto apresentado e todos responderam que não, para ambas as questões.

Para o conjunto de representações visuais, foram apresentados todos os gráficos utilizados e os indicadores que eles representam. Para cada um dos gráficos, pedimos que os professores respondessem se eles conseguiram entender claramente o que estava sendo apresentado e se o gráfico representava de maneira efetiva o indicador pretendido. De modo geral, os 4 professores concordam positivamente em todas as questões, havendo apenas uma diferença em relação ao gráfico utilizado para representar o tempo de conclusão de cada nível, onde um professor se mostrou "indiferente" para as duas questões.

Para a validação da estrutura da Dashboard, pedimos aos professores que respondessem se ela tava bem estruturada e com uma boa distribuição dos gráficos mostrados. Todos os professores responderam positivamente, sendo que 3 concordam totalmente e 1 parcialmente.

Por fim, para o processo de validação da usabilidade da Dashboard, usamos o modelo SUS (System Usability Scale), proposto por Brooke (1986). O SUS é um questionário composto por 10 questões em escala Likert de 5 níveis. Seu resultado é uma pontuação única, usada para medir a usabilidade geral do sistema.

Com base na pontuação alcançada por cada professor (50, 67.5, 82.5 e 97.5 pontos), em uma escalada de 0 a 100, é possível dizer que nossa Dashboard, pode ser considerada de fácil utilização e com baixo nível de inconsistência. Porém, uma seção de entrevistas será conduzida com cada um dos professores participantes, afim de obter sugestões de melhoramento e compreender possíveis dificuldades encontradas.

\section{Conclusões e trabalhos futuros}

Em nosso estudo, ficou claro que o interesse e as expectativas quanto aos jogos educacionais são bastante expressivos. Também pudemos identificar que mesmo diante de alguns desafios encontrados pelos professores no acompanhamento de seus alunos, os jogos têm sido utilizados como alternativa de ensino em diferentes áreas de conhecimento.

O estudo apresentado tem como intuito favorecer o acompanhamento das participações em jogos educacionais e a construção de soluções mais alinhadas com as reais necessidades dos professores. Dessa forma, apresentamos um modelo genérico de acompanhamento de interações e que pode ser aplicado em diferentes tipos de jogos educacionais, para que seja possível representar os indicadores considerados relevantes pelos professores participantes deste trabalho. 
VIII Congresso Brasileiro de Informática na Educação (CBIE 2019)

Anais do XXX Simpósio Brasileiro de Informática na Educação (SBIE 2019)

Espera-se que com as informações levantadas a partir da aplicação do survey e com os conjuntos de indicadores e dados propostos, possamos contribuir tanto com a comunidade de desenvolvimento de jogos, como também com o avanço do estado da arte na área de InfoVis em jogos educacionais, uma vez que são apresentadas as diretrizes para atender as principais necessidades de acompanhamento apontadas pelos professores, tornando mais fácil a aplicação de análise de dados em jogos educacionais.

Como trabalhos futuros, as próximas etapas planejadas para aprimorar este estudo consiste no refinamento da solução proposta, com base nas respostas obtidas no processo inicial de validação e na realização de novos estudos de casos e entrevistas, afim de, alcançar um número maior de professores que utilizam jogos educacionais como alternativa de ensino em suas aulas.

\section{Agradecimentos}

O presente trabalho foi realizado com apoio da Coordenação de Aperfeiçoamento de Pessoal de Nível Superior - Brasil (CAPES) - Código de Financiamento 001

\section{Referências}

ALONSO-FERNANDEZ, C.; CALVO, A.; FREIRE, M.; MARTINEZ-ORTIZ, I.; FERNANDEZ-MANJON, B. Systematizing game learning analytics for serious games. In: IEEE. Global Engineering Education Conference (EDUCON), 2017 IEEE. [S.1.], 2017. p. 1111-1118.

BROOKE, J. System usability scale (sus): a quick-and-dirty method of system evaluation user information. Reading, UK: Digital Equipment Co Ltd, v. 43, 1986.

CARD, S. K.; MACKINLAY, J. D.; SHNEIDERMAN, B. Readings in information visualization: using vision to think. [S.1.]: Morgan Kaufmann, 1999.

CARDOSO, F.; OTSUKA, J. Visualização da informação na análise de dados coletados a partir de jogos: um mapeamento sistemático. In: SBGAMES. Simpósio Brasileiro de Jogos e Entretenimento Digital. [S.1.], 2018.

CARVALHO, E. S.; MARCOS, A. F. Visualização de informação. [S.1.], 2009.

FEW, S. Data visualization for human perception. The Encyclopedia of Human-Computer Interaction, 2nd Ed., The Interaction Design Foundation, 2013.

PRENSKY, M. Aprendizagem baseada em jogos digitais. São Paulo: SENAC, p. 575, 2012.

SERRANO-LAGUNA, Á.; FERNÁNDEZ-MANJÓN, B. Applying learning analytics to simplify serious games deployment in the classroom. In: IEEE. Global Engineering Education Conference (EDUCON), 2014 IEEE. [S.1.], 2014. p. 872-877.

SQUIRE, K. D. Games, learning, and society: Building a field. EDUCATIONAL TECHNOLOGY-SADDLE BROOK THEN ENGLEWOOD CLIFFS NJ-, EDUCATIONAL TECHNOLOGY PUBLICATIONS, v. 47, n. 5, p. 51, 2007. 